\title{
Стандарт качества рутинных методик высокоэффективной жидкостной хроматографии
}

\author{
Е. А. Окунская', К. С. Сычев, к.х.н.' , В. В. Орловский, к.Х.н. ${ }^{2}$
}

УДК 543.544.5.068.7

\begin{abstract}
Обсуждаются основные факторы, сдерживающие рост рынка высокоэффективной жидкостной хроматографии (ВЭЖХ): низкая экономическая эффективность ВЭЖХ-методик и отсутствие отраслевого стандарта качества. Подробно описаны характеристики качественной ВЭЖХХ-методики: правильность, специфичность, робастность, чувствительность, а также экономическая эффективность. Представлен и подробно рассмотрен список из шести основных требований к современным рутинным ВЭЖХ-методикам, который может стать основой стандарта. Приведено несколько прикладных примеров, демонстрирующих основные принципы. Отмечено отрицательное влияние информационного хаоса, когда рекламируются как новые, так и устаревшие продукты, сама реклама не всегда добросовестная, а компании часто противоречат друг другу.
\end{abstract}

Ключевые слова: высокоэффективная жидкостная хроматография, ВЭЖХ-методика, правильность, специфичность, робастность, чувствительность, экономическая эффективность, стандарт

The main factors constraining the growth of the high performance liquid chromatography (HPLC) market are discussed: Iow economic efficiency of HPLC methods and the absence of an industry quality standard. The characteristics of a qualitative HPLC method are described in detail: correctness, specificity, robustness, sensitivity, as well as economic efficiency. A list of six basic requirements for modern routine HPLC methods, which can form the basis of the standard, is presented and discussed in detail. Several application examples are provided to demonstrate the basic principles. The negative impact of information chaos was noted, when both new and outdated products are advertised, the advertising itself is not always fair, and companies often contradict each other.

Keywords: high performance liquid chromatography, HPLC technique, correctness, specificity, robustness, sensitivity, economic efficiency, standard

Статья получена 5.08.2020 Принята к публикации 26.08.2020

\section{Введение}

Любая молодая прикладная дисциплина или производственная технология рано или поздно сталкиваются с «болезнью роста", которая состоит в том,

1 иП Сычев К. С., Орел, sales@hplc.today.

2 Helix Chromatography, Чикаго, США, mail@helixchrom.com. что нет единого понимания сообществом стандарта качества для этой дисциплины (или технологии).

Поскольку любой стандарт менеджмента качества (CMK) неразрывно связан с самим понятием качества, отсутствие ответа на простой вопрос «что такое хорошо, а что такое плохо", по сути, обесценивает любые дальнейшие попытки управления качеством, включая внедрение системы СМК на предприятиях. 
Любые подобные благие намерения закономерно превращаются в бюрократическую бессмыслицу, поскольку не ясны конечная цель преобразований и их механизм.

Современная ВЭЖХ - классический пример молодой прикладной дисциплины. Она прошла совсем небольшой период "взросления": примерно двадцать лет до 80-х годов 20 века - а затем начался период взрывного массового внедрения.

В таких условиях на рынке представлены как наиболее новые, так и крайне устаревшие продукты, а информационное поле заполняется устаревшими прикладными наработками и рекламой. Маркетологическая терминология, по сути, подменяет научную, а маркетинг становится единственной массовой альтернативой знаний на научной базе.

При этом рекламируются как наиболее новые, так и устаревшие продукты, а сама реклама бывает как добросовестной, так и нет. Кроме того, реклама различных компаний часто противоречит друг другу. Фокус маркетинга также часто смещается, что приводит к возникновению новой псевдонаучной терминологии.

Разобраться в этом информационном хаосе бывает непросто даже опытным специалистам, не говоря о значительно большей доле начинающих.

Главным же негативным последствием информационного хаоса можно с уверенностью считать размывание представлений об отраслевом стандарте качества.

\section{Будущее метода}

«Какое ВЭЖХ-решение считать хорошим, а какое плохим?» - вот основной вопрос, от ответа на который зависит будущее метода.

Для корректного ответа на него необходимо, в первую очередь, понимать, что такое «ВЭЖХ-решение».

Вообще, "аналитическое решение" представляет собой комплекс: прибор, методика и расходные материалы. Но у ВЭЖХ есть очень сильная специфика: прибор, по сути, является вспомогательным инструментом, а аналитические характеристики в основном определяются ВЭЖХ-методикой и применяемой ВЭЖХ-колонкой. Тем более, что для рутинных ВЭЖХ-анализов применяют приборы с практически одинаковыми техническими характеристиками.

С качеством колонок, как и с представлениями об их качестве, как ни странно, сейчас больших проблем нет. Несколько упрощая, можно сказать, что новейшие ВЭЖХ-колонки более качественные, и специалисты это понимают.

\section{Качество ВЭЖХ-решений определяется проблемами ВЭЖХ-методик}

И этих проблем очень много. Для начала стоит отметить, что большинство отраслевых ВЭЖХ-методик разработано в 1980-2000-х годах на морально устаревших на данный момент ВЭЖХ-фазах. В результате, самый массовый и "раскрученный" продукт на рынке - несовременные и очень посредственные по качеству колонки, в то время как внедрение новых и качественных сильно пробуксовывает по чисто бюрократическим причинам.

Таким образом, доминирование устаревших методик, по сути, нивелирует позитивный фактор присутствия на рынке новых качественных ВЭЖХ-колонок.

Что касается качества самих методик, то оно фактически раскладывается на два фактора: "пригодность" методики и ее экономическая эффективность.

Более качественная ВЭЖХ-методика должна быть более "пригодной": правильной, специфичной, робастной, чувствительной - а также более экономически эффективной

С понятием пригодности, опять же, больших проблем не возникает: специалисты в целом хорошо понимают какие методики более пригодны. Очевидно, что это методики, которые легко и беспроблемно проходят процедуры валидации и трансфера, обладают большей правильностью и более широкой областью применения.

С экономической эффективностью ВЭЖХ-методик дело обстоит очень плохо. В это сложно поверить, но в течение трех последних десятилетий, то есть половину времени существования метода, она не росла.

Все это не играло большой роли на этапе экспонен ${ }^{-}$ циального роста продаж в 1990-х и 2000-х, но сегодня ситуация на рынке ВЭЖХ очень сложная. Об этом можно судить по изменению объема выручки глобального рынка ВЭЖХ. К сожалению, эти данные не так легко получить из открытых источников, и эти числа различаются.

Тем не менее, можно привести следующие оценки объема рынка ВэЖХ за последние годы (млрд долл.): 4,46 [2] (2017), 3,86 [1] - 4,02 [3] (2018), 4,22 [1] - 4,23 [3] (2019); прогноз на 2020 год (сделан до пандемии): 3,45 [1] - 4,50 [3].

Таким образом, рынок ВэЖХ как минимум стагнирует, если не сокращается. Ни о каком росте в $5 \%$ CACR, который обещают различные аналитические агентства, пока не может быть и речи. 
Причина этого достаточно очевидна. Драйвером роста рынка ВЭЖХ является именно ВЭЖХ для рутинных анализов (для исследований сейчас применяют ВЭЖХ-МС, то есть, по сути, исследования - это рынок MC). Стагнация сектора рутинной ВЭЖХ говорит о том, что все профильные лаборатории уже более или менее обеспечены базовым ВЭЖХ-оборудованием, и серьезного роста здесь уже не достичь.

Другими словами, эпоха простых продаж и экстенсивного роста закончилась. Чтобы рынок рос, необходимо выходить на более массового потребителя, но с текущими подходами крупных производителей к маркетингу и продажам это просто невозможно.

Массовый потребитель, вследствие меньшего профессионализма, предъявляет более высокие требования к качеству приложений (здесь - к надежности ВЭЖХ-методик, простоте процедуры анализа), к их экономической эффективности (высокая скорость, низкие затраты), удобству и автономности всего решения в целом и, самое главное, к общей стоимости решения. Чтобы выполнить все эти требования, необходимо работать над увеличением качества ВЭЖХ-методик, и прежде всего - их экономической эффективностью.

Но чтобы работать над качеством, нужно знать ответ на вопрос "какое ВЭЖХ-решение считать хорошим, а какое - плохим" - и мы вернулись к тому, с чего начали: дальнейшее развитие невозможно без понимания сообществом единого стандарта качества ВЭЖХ-методик.

Таким образом, основными факторами, сдерживающими рост рынка ВЭЖХ, являются низкая экономическая эффективность ВЭЖХ-методик и отсутствие отраслевого стандарта качества, который бы позволял работать в направлении ее повышения.

Задача этой статьи состоит в том, чтобы на нескольких прикладных примерах продемонстрировать ряд базовых принципов, которые могут стать основой подобного отраслевого стандарта качества.

\section{Оборудование}

\section{и хроматографические колонки}

- Жидкостной хроматограф ЕСОМ (ЕСОМ, Чехия), укомплектованный насосом ЕСР2010Н, диодноматричным детектором ECDA2800 и термостатом колонок ЕСО2099.

- ВЭЖХ-колонка Coresep 100, 150×4,6, 2,6 мкм (Helix Chromatography, США);

- ВЭЖХ-колонки IBSpharm AH-RP18, IBSpharm AHS, 250×4,6, 3 мкм (IBS, Эстония);
- ВЭЖХ-колонка Cosmosil 5PYЕ, 250×4,6, 5 мкм (Nacalai Tesque, Япония);

- ВЭЖХ-колонка Ace Excel C18-PFP, 250×4,6, 3 мкм (VWR, Великобритания).

\section{Экспериментальные результаты и обсуждение}

По нашему мнению, качественная современная ВЭЖХ-методика для рутинных измерений должна удовлетворять шести требованиям.

1. Рутинная ВЭЖХ-методика должна быть как можно более специфичной, то есть основанной на ВЭЖХ-приложении с высокой специфичностью разделения и / или высокой специфичностью детектирования.

2. Эффективность и симметрия пиков (для каждого конкретного приложения) должны соответствовать таковым для лучших образцов коммерческих неподвижных фаз на рынке, а не просто “быть достаточными", “средними" или, тем более, "ниже среднего".

3. Время анализа по возможности не должно превышать 15-20 мин для насосных систем 400 бар, или 5-10 мин для насосных систем 800-1500 бар.

4. ВЭЖХ-разделение должно быть изократическим.

5. Подвижная фаза по возможности не должна содержать модифицирующих добавок, особенно органических. Буферы для подвижной фазы должны быть как можно менее агрессивными.

6. Условия проведения хроматографического анализа должны по возможности обеспечивать наиболее простую, быструю и недорогую подготовку пробы.

Несколько упрощая, можно сформулировать так: чем большим числом преимуществ (приведенных в этом списке) обладает рутинная ВЭЖХ-методика, тем она качественнее.

Такой простой подход, скорее всего, не годится для сравнения нескольких хороших методик. С другой стороны, он гарантированно позволяет отсеивать полностью непригодные и экономически неэффективные - что уже является шагом вперед.

Теперь - подробнее о каждом из перечисленных требований.

1. Фокус на специфичности

Специфичность - это валидационная характеристика методики, от которой напрямую зависит правильность определения. При этом специфичность 
полностью определяется условиями хроматографического определения, то есть применяемой ВЭЖХ-колонкой, составом подвижной фазы и условиями детектирования.

Таким образом, чем специфичнее ВЭЖХ разделение, тем правильнее результаты определения по соответствующей методике на одном образце, или же шире область применения данной ВЭЖХ-методики при фиксированной правильности.

Оба практических следствия более высокой специфичности означают большую пригодность соответствующих методик, а второе следствие (более широкая область применения) означает еще и более высокую экономическую эффективность.

Специфичность можно увеличить двумя путями. Проще всего усилить специфичность детектирования, но сделать это можно далеко не всегда.

Более универсальный подход состоит в увеличении специфичности хроматографического разделения. В рамках обращеннофазовой хроматографии (RP HPLC) можно применять более специфичные смешанные обращеннофазовые режимы (RP/IC, RP/CT, RP/HILIC), или можно изначально применять более специфичные режимы, такие как гидрофильный (HILIC), или ионный (IC).

Чтобы не "потерять" удачные решения при разработке новых приложений для рутинного анализа, в первую очередь подбирают наиболее избирательные условия детектирования, а затем - условия разделения, начиная с самых специфичных режимов, и заканчивая неспецифичными (например, классической обращеннофазовой хроматографией).

Характерным признаком специфичных разделений является их высокая межгрупповая селективность. Другими словами, применение хроматографических условий, в которых окна элюирования различных классов веществ не пересекаются, снижает вероятность наложения сигналов контаминантов на сигналы целевых соединений и, таким образом, способствует увеличению пригодности методик.
Наилучшие же с экономической точки зрения рутинные разделения получаются, когда удается добиться сочетания высокой межгрупповой селективности с умеренной (то есть не низкой, но и не высокой) внутригрупповой селективностью.

В этих случаях соединения одного химического класса элюируются достаточно компактными группами, и специфичность разделения комбинируется с высокой скоростью анализа. К примеру, такие решения идеально подходят для определения родственных примесей.

Примером также может служить HILIC/WCX изократическое разделение антигистаминных фармацевтиков в комбинированных препаратах (рис. 1). Целевые соединения хорошо отделяются даже от близких по структуре компонентов матрицы (метамизол, декстрометорфан), не говоря уже о нейтральных (парацетамол) и кислых (ксантановая камедь, напроксен) контаминантах. При этом для

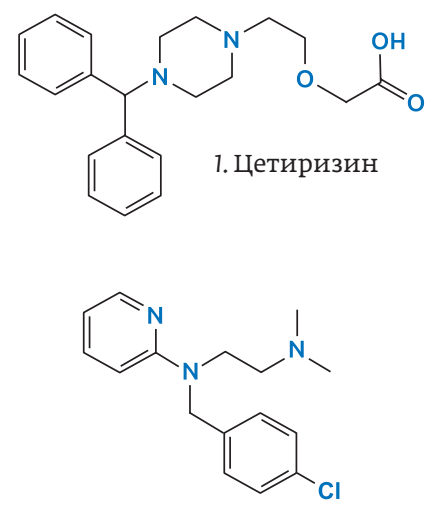

2. Хлоропирамин

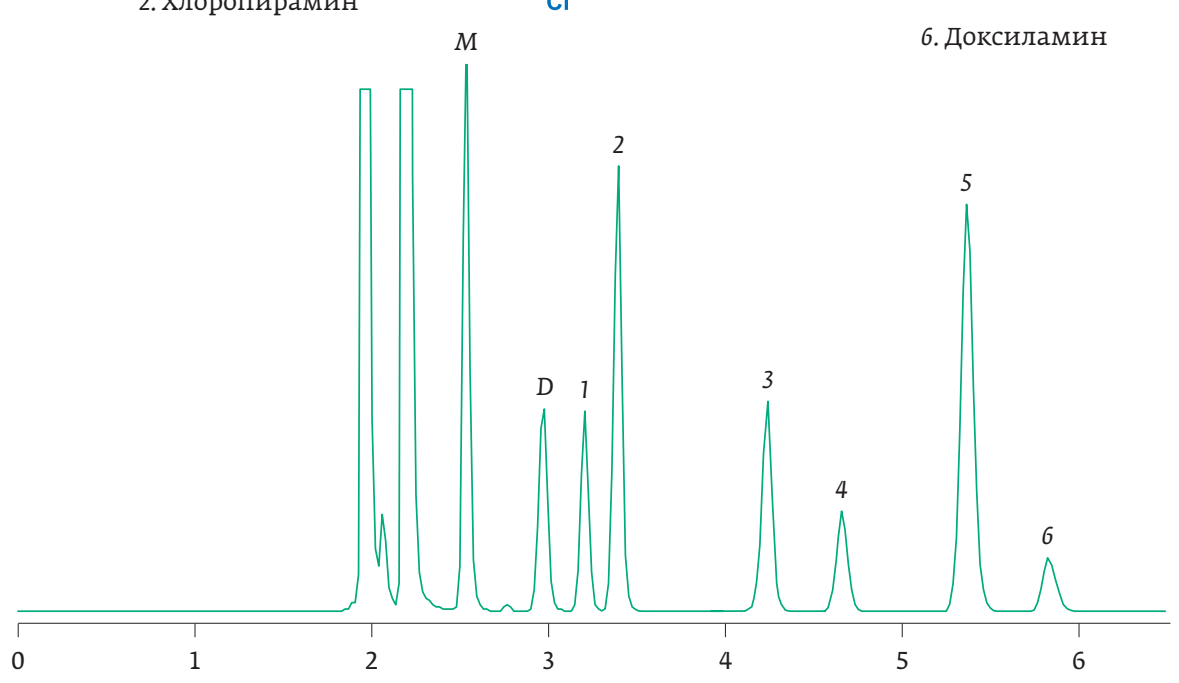

Puc. 1. Специфичное определение шести антигистаминных фармацевтиков в смеси шести комбинированных препаратов. Детектирование: УФ 260 нм. ВЭЖХ-колонка: IBSpharm AHS, 250 × 4,6; 3 мкм. Идентифицированные компоненты матрицы: $M$ - метамизол, D - декстрометорфан 
разделения шести соединений оказывается достаточно всего 1,5 единиц $k^{\prime}$ (разделение происходит в интервале $\left.k^{\prime}=0,5-2\right)$, что на практике может быть реализовано за 6 мин при давлении порядка 250 атм.

\section{2. Высокая эффективность и симметрия пиков}

Большая проблема, не позволяющая добиться заметного прогресса в области разработки рутинных ВЭЖХ-методик, связана с широким и систематическим применением на практике морально устаревших и просто некачественных неподвижных фаз для ВЭЖХ.

Отличительные признаки таких фаз - низкая эффективность и асимметрия хроматографических пиков для органических оснований и соединений, обладающих хелатирующей способностью.

Так, в "реальных рабочих условиях" эффективность в несколько тыс. т. т. при асимметрии вплоть до 1,5 (ЕР) до сих пор считается нормой в фармацевтике - хотя это совершенно ненормально. Даже при не очень высокой сложности разделений такие низкие значения эффективности могут стать основной причиной низкой правильности измерений.

Основной технической причиной пониженной симметрии и эффективности хроматографических пиков многих основных соединений является низкая чистота исходного силикагеля, который был применен для синтеза неподвижной фазы. Для работы необходимо применять лишь фазы на основе особо чистых силикагелей (extra-pure silica, >99,99\% или $>99,999 \%$ ).

На рис. 2 приведен пример ВЭЖХ обращеннофазового разделения основных соединений различных химических классов (антигипертензивных фармацевтиков) без применения каких-либо модифицирующих добавок в составе подвижной фазы.

Как видно из разделения, все соединения элюируются с симметрией около 1,0 и практически одинаковой (и при этом предельно высокой) эффективностью 30 000-40 000 (удельная эффективность 120-160 т. т. / М для 3 мкм адсорбента соответствует ВЭТТ 8,3-6,3 мкм, то есть близко к предельному значению $2 d_{p}=6$ мкм). Такой результат не является экстраординарным - просто для разделения применяется обращенная фаза на основе высокочистого силикагеля.

По нашему мнению, следующие значения эффективности можно считать нормой для современных рутинных ВЭЖХ-решений (для насосных систем 400-600 бар):

- ионный (IC) режим: 5000-25000;

- гидрофильный (HILIC) режим: 10 000-40 000;

- обращенно-фазовый режим (RP): 15 000-60 000;

- нормально-фазовый режим (NP): 15000-80 000.

\section{3. Время анализа}

Приведенные значения эффективности вполне достаточны для выполнения разделений средней сложности, требующие величин пиковой емкости порядка 50-70. Более того, для этого вполне 
достаточно давления не более 300 бар и времени не более 15 мин. К примеру, для насосных систем 400 бар и вязкости, соответствующей смеси водаацетонитрил $1: 1$, для рутинных приложений оптимальны следующие два типоразмера ВЭЖXколонок:

1. $250 \times 4,6$ колонки с 3 мкм полностью пористыми частицами при 1,0 мл/ мин; $k^{\prime}$ 0,5-5: $t_{\mathrm{R}}$ (время анализа $)=$ $=250 \cdot 6 / 1 \cdot 0,01 \approx \mathbf{1 5 , 0 ~ м и н ; ~}$

$n$ (пиковая емкость $)=$ $=1+\sqrt{40000} / 4 \cdot \ln (4) \approx 70 ;$

2. $250 \times 4,6$ колонки с 3,5 мкм полностью пористыми частицами при 1,5 мл/ мин; $k^{\prime}=0,5-5: t_{\mathrm{R}}=250 \cdot 6 / 1,5 \cdot 0,01 \approx$ $\approx 10,0$ мин; $n=1+\sqrt{25000} / 4 \cdot \ln (4) \approx 55$.

Пример изократического разделения на ВЭЖХ-системе 400 бар с оценочной пиковой емкостью n=60-70 за 15 мин (условие 1) приведен на рис. 3.

Применение насосных систем 600 бар, которые уже являются современным стандартом, позволяют достичь еще большей пиковой емкости за приемлемое время, либо еще большей производительности при той же сложности разделения:

3. $250 \times 4,6$ колонки с 2,7 мкм поверхностнопористыми частицами при 1,25 мл / мин; $k^{\prime} 0,75-6: t_{\mathrm{R}}=250 \cdot 7 / 1,25 \cdot 0,01 \approx 14,0$ мин; $n=1+\sqrt{60000} / 4 \cdot \ln (4) \approx 82$;

4. $250 \times 4,6$ колонки с 2,7 мкм поверхностнопористыми частицами при 1,25 мл / мин; $k^{\prime} 0,75-3: t_{\mathrm{R}}=250 \cdot 4 / 1,25 \cdot 0,01 \approx \mathbf{8 , 0}$ мин; $n=1+\sqrt{60000} / 4 \cdot \ln (2,3) \approx 52$.

Таким образом, даже при весьма солидной пиковой плотности $n=60-70$ время анализа при правильно подобранном удерживании не превышает 15-20 мин для насосных систем 400 бар, или 10-15 мин для 600 бар-систем.

\section{4. Изократический режим элюирования}

Еще одна массовая вынужденная ошибка, приводящая к низкому качеству рутинных ВЭЖХ-разделений, состоит в широком использовании градиентного элюирования. Вынужденный характер этой ошибки обусловлен неоправданно широким применением классической обращенно-фазовой хроматографии.

Обращенно-фазовая ВэЖХ - это наиболее неспецифичный подход, при котором низкая межгрупповая и очень высокая внутригрупповая селективность.

Необходимость применения градиентных условий элюирования часто бывает вызвана именно избыточной внутригрупповой селективностью: соединения даже с однотипной химической структурой так сильно разделяются, что не могут быть элюированы с колонки за приемлемое время в изократических условиях. Другими словами, градиентом состава подвижной фазы банально уравновешивают избыток внутригрупповой селективности применяемой обращенной фазы .

Чем же так плохи градиентные условия для рутинных ВЭЖХ-разделений? Главный недостаток связан 
с тем, что в реальности градиентные методики бывает очень трудно валидировать, а еще большие сложности доставляет их трансфер.

"Трудно» - это значит с большими затратами времени, реагентов, труда специалистов. Причем все это при посредственном, ненадежном результате, то есть, как правило, низкой робастности (так, селективность зависит от конструкции прибора: жидкостной системы, термостата и теплообменника) и нередко недостаточно высокой специфичности (уменьшение селективности разделения до приемлемого уровня по краям градиента может привести к падению селективности разделения ниже приемлемого уровня на подъеме градиента).

Также нередки случаи, когда в результате плохо подобранного градиентного профиля быстро выходят из строя элементы насосной и жидкостной системы (забиваются и залипают клапаны, залипают плунжера, забиваются входные фриты колонок). В результате, градиентные методики обладают очень посредственной пригодностью и низкой экономической эффективностью.

Для рутинных ВЭЖХ-разделений лучше применять специфичные изократические ВЭЖХ-решения .

В крайнем случае, когда необходимо провести сложное разделение в условиях избыточной внутригрупповой селективности, вместо градиентного элюирования следует применять неподвижные фазы с наименьшей внутригрупповой селективностью в изократическом режиме.

В случае обращенно-фазовой хроматографии таковыми являются неподвижные фазы с минимальной метиленовой селективностью; как правило, такие обращенные фазы работают в смешанных обращенно-фазовых режиmax: RP/HILIC, RP/CT, RP/IC.

На рис. 4 приведен пример изократического разделения смеси в смешанном RP/CT режиме, которое на классической с18-фазе можно провести лишь с применением градиентного элюирования с крутым подъемом градиента.
5. Отсутствие модифицирующих добавок

Одна из отличительных черт устаревших ВЭЖХ-методик - широкое применение органических модифицирующих добавок в составе подвижной фазы.

Модифицирующие добавки в подвижные фазы были всегда мерой вынужденной, своего рода наименьшим из зол. Применяли их в те времена, когда не существовало современного разнообразия различных химий неподвижных фаз, а доступные были крайне низкого качества (в первую очередь, плохого качества был исходный силикагель).

В настоящее время необходимость таких подходов полностью отпала, но их часто применяют "по инерции", не задумываясь, или же сознательно, чтобы "обеспечить преемственность методик". Ничего хорошего, впрочем, перенять у методик с динамическим модифицированием нельзя. А вот типичные недостатки таких подходов - всегда при них.

Все подобные методики очень плохо воспроизводятся, а их робастность оставляет желать лучшего.<smiles>CNC[C@H](O)c1cccc(O)c1</smiles>

1. Фенилэфрин<smiles>CC(=O)Nc1ccc(O)cc1</smiles>

2. Ацетаминофен<smiles>COc1ccc2c3c1O[C@H]1[C@@H](O)C=CC[C@]31[C@@H]1CCN(C)[C@@H]2C1</smiles>

5. Кодеин<smiles>Cn1c(=O)c2c(ncn2C)n(C)c1=O</smiles>

7. Кофеин<smiles>CNC(C)[C@H](O)c1ccccc1</smiles>

3. Псевдоэфедрин<smiles>Cc1c(N(C)CS(=O)(=O)O)c(=O)n(-c2ccccc2)n1C</smiles>

4. Метамизол<smiles>COc1ccccc1OCC(O)CO</smiles>

6. Гуафенезин

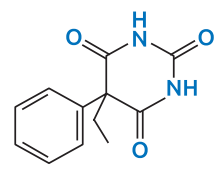

8. Фенобарбитал
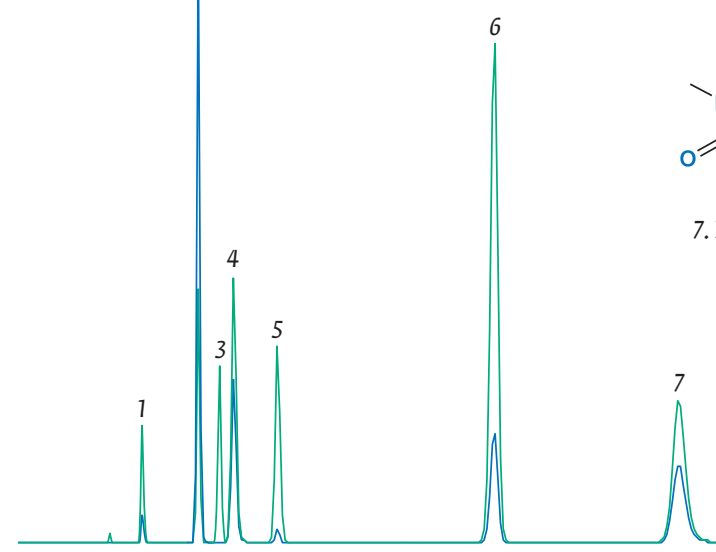

30

Puс. 4. Изократическое разделение восьми компонентов противопростудных лекарственных средств. Детектирование: УФ 215 нм (-), 280 нм (一). ВЭЖХ-колонка: Cosmosil 5PYE, 250 ×4,6; 5 мкм 
Специфичность - также их уязвимое место, поскольку системы с динамическим модифицированием могут генерировать много системных сигналов, усложняющих разделение. Кроме того, качественные органические добавки весьма дороги, особенно при рутинном применении, а динамически модифицированную ВЭЖХ-колонку можно применять только для одного определенного анализа, в комбинации с одной соответствующей подвижной фазой.

Среди подходов с динамическим модифицированием наиболее широко известна ион-парная обращенно-фазовая ВЭЖХ, где “сильные» ион-парные реагенты (С6-C10 алкилсульфонаты, додецилсульфат, тетрабутиламмоний), по сути, применяются для получения ионных неподвижных фаз из обращеннофазовых. Никакой нужды делать так сейчас нет, все подобные разделения можно осуществить на подходящих современных ионных фазах, или же решить все эти задачи другими способами.

Диэтиламин и триэтиламин (ТЭА) ранее широко применяли для улучшения формы пиков органических оснований - когда, опять же, качество силикагелей были крайне низким. Сейчас в этом также нет никакого смысла.

ТЭА, ТФУ, бутилсульфонат иногда применяют как "слабые" ионпарные добавки для исправления небольших огрехов в разделении (например, не до конца разделенных пар), но это больше напоминает "заплатку" на неработающий метод, чем отдельный серьезный подход.

Остальные органические добавки применяют для создания буферной емкости: формиат, ацетат, цитрат, ЭДТА. Здесь наиболее опасными добавками являются цитрат и ЭДТА. Они плохо растворимы в любых средах и легко откладываются на различных элементах жидкостной системы, включая клапаны и плунжеры насосов.

Сегодня для ряда случаев имеет смысл использовать такие органические добавки, как формиат аммония и муравьиная кислота. В первую очередь, их применяют в полностью безводных средах или там, где доля водной части минимальна (например, менее 15\% в ацетонитриле, или менее $30 \%$ в метаноле).

0
ВЭЖХ-приложения, в которых фигурируют органические добавки, не следует копировать; напротив, желательно переделывать их таким образом, чтобы исключить применение добавок.

На рис. 5 приведено разделение катехоламинов с подвижной фазой на основе водного раствора кислого фосфата аммония, в то время как "классическая" методика предписывает применение цитратного буфера с добавкой ЭДТА. Очевидно, что ни цитрат, ни ЭДТА не нужны для качественного ВЭЖХ-разделения катехоламинов.

\section{6. Минимальная подготовка пробы}

Как хорошо известно, самая лучшая подготовка пробы - это ее отсутствие. Тактика dilute-and-shoot (растворил и инжектировал) абсолютно выигрышна, причем как с точки зрения экономической эффективности, так и простоты исполнения - фактора, критичного для массового потребителя .

Следуя этой логике при разработке рутинного метода, в первую очередь следует выбирать те решения, которые дают минимальную подготовку пробы<smiles>NC[C@@H](O)c1ccc(O)c(O)c1</smiles>

1. Норэпинефрин<smiles>COc1cc([C@@H](O)CN)ccc1O</smiles>

4. Норметанефин<smiles>CNC[C@H](O)c1ccc(O)c(O)c1</smiles>

2. Эпинефрин<smiles>CNC[C@H](O)c1ccc(O)c(OC)c1</smiles>

5. Метанефрин<smiles>NCCc1ccc(O)c(O)c1</smiles><smiles>NCCc1c[nH]c2ccc(O)cc12</smiles>

6. Сератонин

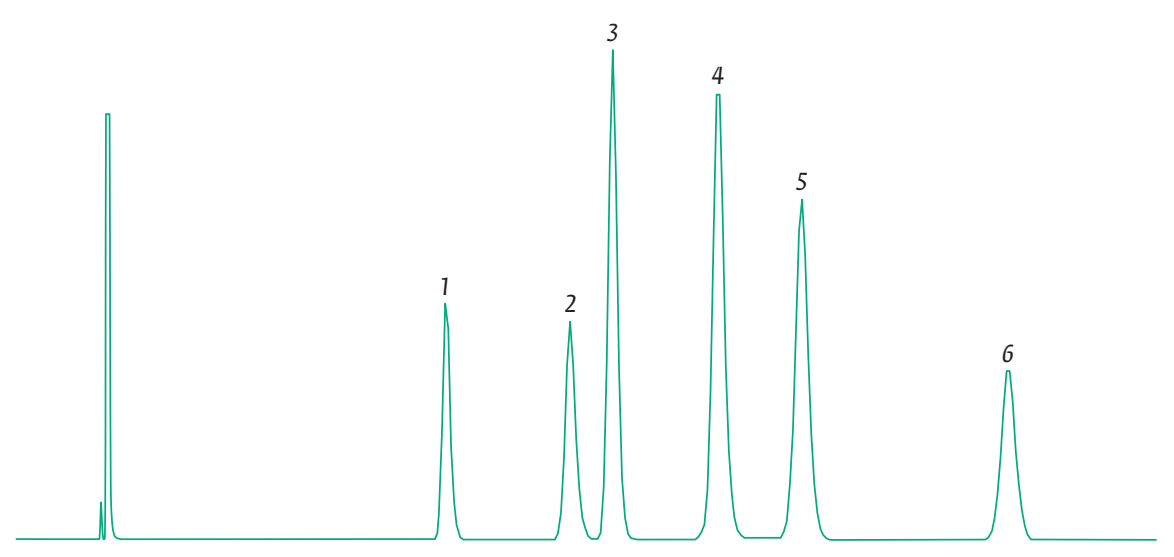

5

Рис. 5. Изократическое разделение пяти катезоламинов и серотонина. Детектирование: УФ 215 нм. ВЭЖХ-колонка: Coresep 100, $150 \times 4,6 ; 2,7$ мкм 
в сочетании с высокой специфичностью определения. К счастью, эти два ключевых требования к рутинным ВЭЖХ-разделениям достаточно часто идут рука об руку, и вероятность встретить такое удачное решение среди всех возможных довольно высока.

\section{Вывод}

Основными факторами, сдерживающими рост рынка ВЭЖХ, являются низкая экономическая эффективность ВЭЖХ-методик и отсутствие отраслевого стандарта качества, который бы позволял работать в направлении ее повышения.

Список из шести основных требований к современным рутинным ВЭЖХ-методикам, предложенный в статье, может стать основой такого стандарта.

\section{Литература / References}

1. Global High Performance Liquid Chromatography (HPLC) Market-Forecast and Analysis (2019-2026), by Type, Process Component, Technology, End-User and Region. https://www.maximizemarketresearch.com/market report/global-high-performance-liquid-chromatographymarket/33778/. (Дата обращения 01.08.2020)

2. High-Performance Liquid Chromatography (HPLC) Market Forecasts from 2017 to 2022.

https://www.researchandmarkets.com/reports/4377945/ high-performance-liquid-chromatography-hplc. (Дата обращения 01.08.2020).

3. High-performance Liquid Chromatography (HPLC) Market by Product (Instruments (Systems, Detectors), Consumables (Columns, Filters), and Accessories), Application (Clinical Research, Diagnostics, Forensics), Region-Global Forecast to 2025.

https://www.marketsandmarkets.com/Market-Reports/ hplc-market-117899209.html. (Дата обращения 01.08.2020).

\section{НОВЫЙ КАТАЛИЗАТОР ЭФФЕКТИВНО ПРЕВРАЩАЕТ ДИОКСИД УГЛЕРОДА В ПОЛЕЗНОЕ ТОПЛИВО И ХИМИЧЕСКИЕ ВЕЩЕСТВА}

Содержание углекислого газа в атмосферепродолжает расти, аученые ищут новые способы расщепления молекул $\mathrm{CO}_{2}$ для производства полезных видов топлива на основе углерода. Группа исследователей из Университета Брауна нашла способ тонкой настройки медного катализатора для получения сложных углеводородов, известных как продукты Q-plus из $\mathrm{CO}_{2}$ с высокой эффективностью.

В исследовании, опубликованном в Nature Communications, сообщается о катализаторе, который может производить соединения Q2-plus с фарадеевской эффективностью (показатель того, насколько эффективно используется электрическая энергия для преобразования диоксида углерода в продукты химической реакции) до $72 \%$. По словам исследователей, это намного выше известных эффективностей других катализаторов для получения Q2-plus, а процесс его изготовления можно легко довести до промышленного уровня, что открывает перспективы использования катализатора в крупномасштабныхпроектахпо переработке $\mathrm{CO}_{2}$. «В литературе были сообщения о всевозможных видах обработки меди, спомощью которых можно было бы производить Q-plus с широким диапазоном эффективности», - сообщает Тайхас Палмор, профессор инженерных наук из Брауна.

В последние годы были достигнуты большие успехи в разработке медных катализаторов, которые могут производить одноуглеродные молекулы. Например, недавно был разработан катализатор из вспененной меди, который может эффективно производить муравьиную кислоту - важный одноуглеродный химический продукт. Однако, растет интерес к реакциям, приводящим к образованию Q-plus-продуктов.

Предыдүщие исследования показали, что галогенирование меди (реакция, при которой поверхность меди покрывается атомами хлора, брома или йода в присутствии электрического потенциала) повышает селективность катализатора в отношении C2-plus-продуктов. Были проведены исследования с множеством различных методов галогенирования с целью определения, какие галогеновые элементы и электрические потенциалы дают катализаторы с наилучшими характеристиками в реакциях перехода $\mathrm{CO}_{2}$ В $\mathrm{O}$-plus. Обнаружено, что

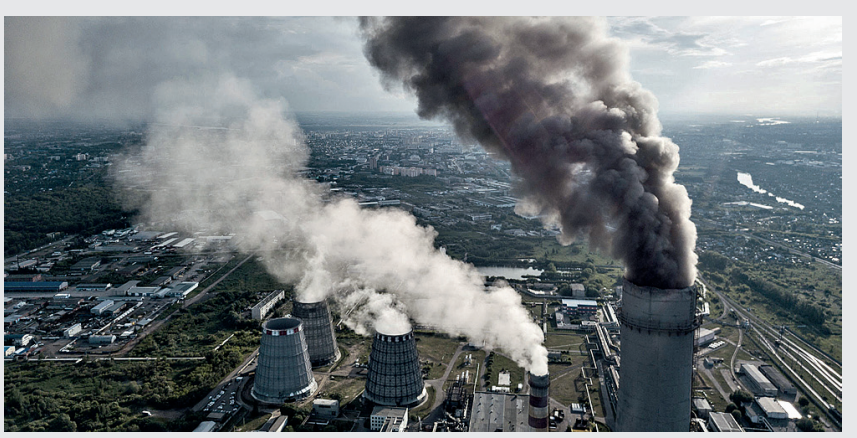

оптимальные препараты могут давать фарадеевскую эффективность от 70,7 до $72,6 \%$, что намного выше, чем у любого другого медного катализатора.

Катализаторы с наивысшей эффективностью имели большое количество поверхностных дефектов - крошечных трещин и щелей на галогенированной поверхности. Они имеют решающее значение для реакций үглерод-үглеродного взаимодействия. Эти дефектные участки являются ключом кдостижению высокой селективности катализаторов по отношению к этилену и C2-plus-продуктам, которые можно использовать для производства пластмасс.

В перспективе такой катализатор поможет в крупномасштабной утилизации $\mathrm{CO}_{2}$. Идея состоит в том, чтобы улавливать $\mathrm{CO}_{2}$, производимый промышленными предприятиями, такими как электростанции, цементные заводы, - непосредственно из воздуха, и преобразовывать его в другие полезные углеродные соединения. Для этого требуется эффективный и недорогой катализатор, который легко производить и регенерировать для работы в промышленных масштабах. Полученный катализатор имеет очень хорошие перспективы промышленного внедрения. Разработанный метод позволяет получить катализатор практически любого размера.

По мamepuanam https://www.sciencedaily.com 
Всероссийской конференции

«Аналитическая хроматография и капиллярный электрофорез»

с международным участием

Место проведения: Оздоровительный комплекс "Орбита»,

с. Ольгинка, Туапсинский район, Краснодарский край.

\section{ТЕМАТИКА КОНФЕРЕНЦИИ}

- Теория и физико-химические основы хроматографических и электрофоретических процессов;

- селективность и эффективность разделения методами хроматографии и капиллярного электрофореза;

- состояние и перспективы инструментального обеспечения хроматографических и электрофоретических исследований;

- метрологическое обеспечение, хемометрика и обработка данных в хроматографии и капиллярном электрофорезе;

- сорбенты и материалы для хроматографии и электрофоретического анализа;

- практическое использование методов хроматографии и капиллярного электрофореза.

Программа конференции включает пленарные заказные доклады, устные и стендовые сообщения. В качестве пленарных докладчиков планируется привлечь ведущих ученых в данной области. Рабочий язык конференции - русский.

\section{Организационный комитет}

- Золотов Ю.А., академик РАН - Почетный Председатель

- Шпигун О.А., член-корр. РАН - сопредседатель

- Колотов В.П., член-корр. РАН - сопредседатель

- Буряк А.К., член-корр. РАН - зам. председателя

- Темердашев 3.A., д.Х.н. - зам. председателя

- Татаурова О.Г., К.Х.Н. - ученый секретарь

- Грузнов В.М., Д.Т.Н.

- Карцова Л.А., д.Х.Н.

- Киселева Н.В., К.Х.н.

- Красиков В.Д., Д.Х.Н.

- Курганов А.А., А.Х.н.

- Пирогов А.В., д.Х.н.

- Платонов И.А., А.Т.Н.

- Ревельский А.И., д.Х.н.

- Рыбальченко И.В., д.Х.н.

- Селеменев В.Ф., д.Х.н.

- Смоленков А.Д., Д.Х.н.

- Спиваков Б.Я., член-корр. РАН

- Темердашев А.3., к.Х.н.

- Цизин Г.И., д.х.н.

- Яшкин С.Н., Д.Х.н. 